normal on serial testing. A review of the recent medical literature yielded only one case in which hypercortisolism disguised symptoms and signs of an abdominal surgical crisis in a patient who died of an unrecognized gallbladder perforation. ' Presumably in both these cases the welldocumented anti-inflammatory and immunosuppressive effects of hypercortisolism ${ }^{2,3}$ masked the development of infection and reduced the host's response once it has become established. Cushing's syndrome is known to predispose to opportunist infection, in the past up to $46 \%$ of patients with Cushing's syndrome died of sepsis, many following major surgery. ${ }^{4}$ In the modern era opportunist infection is less commonly seen. This case illustrates the need for continuing vigilance in patients with Cushing's syndrome. It reaffirms the commonly held belief that hypercortisolism, whether due to Cushing's syndrome or exogenous steroids, can mask severe abdominal pathology.

\section{Martinez ${ }^{1}$ \\ S.M. Orme \\ A. Chalmers ${ }^{1}$ \\ P.E. Belchetz \\ Departments of Endocrinology and ${ }^{1}$ Radiology, \\ The General Infirmary at Leeds, Great George Street, Leeds LS1 3EX, UK.}

\section{References}

1. Graham, B.S. \& Tucker, W.S. Opportunist infections in endogenous Cushing's syndrome. Ann Intern Med 1984, 101: 334-338.

2. Fauci, A.S., Dale, D.C. \& Balow, J.E. Glucocorticoid therapy: mechanisms of action and clinical considerations. Ann Intern Med 1976, 84: 304-315.

3. Bondy, P.K. Disorders of the adrenal cortex. In: Wilson, J.D. \& Foster, D.W. (eds) Williams Textbook of Endocrinology, 7th ed. W.B. Saunders, Philadelphia, 1985, pp. 816-890.

4. Plotz, C.M., Knowlton, A.I. \& Ragan, C. The natural history of Cushing's syndrome. Am J Med 1952, 13: 597-614.

\section{Trimethoprim-induced vasculitis}

Sir,

Adverse drug reactions associated with co-trimoxazole may be caused by either trimethoprim or sulphamethoxazole. However, the skin rashes are so similar to those caused by sulphonamides alone that most are attributed to these. ${ }^{1}$ We report a case of vasculitis which was induced by trimethoprim.

An 83 year old woman was admitted with severe anaemia ( $\mathrm{Hb} 3.8 \mathrm{~g} / \mathrm{dl}$ ) secondary to vitamin $\mathbf{B}_{12}$ deficiency, attributed to a previous vagotomy and pyloroplasty and a coliform septicaemia secondary to a urinary tract infection. She was treated with intravenous cefuroxime for 11 days. She then suffered a right cerebro-vascular accident.

Following this she made a good recovery. During her rehabilitation, 2 months later, she developed a further urinary tract infection. A mid-stream urine grew coliforms, which were sensitive to trimethoprim, but resistant to ampicillin and nalidixic acid. She was given a week's course of trimethoprim $200 \mathrm{mg}$ twice daily. Nine days later she developed a florid leucocytoclastic vasculitis on her hands, tongue, shins and feet. This was associated with acute arthritis of her interphalangeal joints and haematuria. Previous urinalysis for blood had been negative.

Her daughter then remembered that her mother had had a similar rash 2 years previously when she had been treated with co-trimoxazole for a urinary tract infection. A case summary was obtained and this confirmed that our patient had had a vasculitis, secondary to co-trimoxazole.

Other causes of a vasculitic rash were looked for: a full blood count showed a white cell count of $3.3 \times 10^{9} / 1$, haemoglobin of $14.9 \mathrm{~g} / \mathrm{dl}$ and platelets of $120 \times 10^{9} / 1$. Blood cultures, rheumatoid factor and auto-antibodies were negative. Routine viral titres showed no recent infection. Serum electrophoresis showed a double $\mathbf{M}$ band in the gamma region but there was no Bence-Jones protein. Serum complement and cryoglobulins were in the normal ranges. An ultrasound of her abdomen was normal.

She was treated with hydrocortisone $(200 \mathrm{mg} 4$ times daily) and intravenous antibiotics (to cover for a possible diagnosis of endocarditis). She responded dramatically to treatment over a week but developed a chest infection and died 4 weeks later.

We are convinced that the vasculitis was caused by trimethoprim because of the recurrence of the rash on subsequent exposure and because of the lack of another explanation. The Committee on the Safety of Medicines has a number of reports of various types of rashes being associated with the use of trimethoprim. These include maculo-papular, morbilliform, erythematous, angioderma and purpuric. There was only one previous reported case of a vasculitis from Holland (unpublished, Wellcome Foundation Ltd files) which was described as purpuric and is distributed over the trunk and lower limbs.

Patients who have adversely reacted to co-trimoxazole should receive neither trimethoprim nor sulphamethoxazole in future.

Richard I.G. Holt

Shah B.J. Ebrahim

Department of Health Care of the Elderly, The Royal London Hospital (Mile End), Bancroft Road, London EI 4DG, UK.

\section{References}

1. Weinstein, L., Madoff, M.A. \& Samet, C.A. The sulfonamides. $N$ Engl J Med 1960, 263: 793-800, 842-849, 900-907.

\section{Listeria rhombencephalitis in a previously healthy adult}

Sir,

We report a case of listeria rhombencephalitis (brain stem encephalitis) in a healthy adult.

An 85 years old previously well woman was admitted with a 10 day history of right seventh cranial nerve paresis. She was pyrexial and slightly confused. The neurological examination revealed nystagmus towards 
the left with complete right facial palsy but no neck stiffness.

Investigations showed a white cell count of $20.6 \times 10^{9} / 1$, with $90 \%$ neutrophils. A computed tomographic (CT) scan was entirely normal. However, the patient's clinical condition rapidly deteriorated. She developed marked neck stiffness, became drowsy and her temperature increased to $40^{\circ} \mathrm{C}$. A lumbar puncture showed 100 white cells $/ \mathrm{mm}^{3}$ predominantly lymphocytes with normal protein concentration. No organism was found on Gram staining. A tentative diagnosis of meningoencephalitis was made and the patient was commenced on acyclovir, ampicillin and gentamicin. C-reactive protein on admission was $195 \mathrm{mg} / \mathrm{l}$ (normal $<6 \mathrm{mg} / \mathrm{l}$ ).

Four days after admission Listeria monocytogenes was isolated from the cerebrospinal fluid (CSF) culture. The patient remained drowsy and continued to deteriorate. Chloramphenicol was added to the drug regime. A repeat CT scan was again normal.

Four weeks after admission the patient developed acute renal failure complicated by disseminated intravascular coagulation and died. A post-mortem examination revealed necrotic areas in the pons and medulla on the right side of the brain but no other pathology was found.

L. monocytogenes is a Gram-negative organism which enters the body through the consumption of contaminated food. It mainly effects pregnant women, immunocompromised patients or the elderly. The most common central nervous system manifestation of $L$. monocytogenes is meningitis. Eck reported the first case of brain stem encephalitis in 1957.' Since then a few cases have been reported and notably most of these patients were previously in good health. ${ }^{2}$

The clinical presentation of rhombencephalitis shows a unique bimodal pattern. ${ }^{2-3}$ The prodromal phase of fever, malaise, headache, vomiting and leucocytosis usually precedes the neurological event by $4-10$ days. This is followed by sudden appearance of pontomedullary dysfunction with cranial nerve palsy, however, nuchal rigidity is a rare occurrence. The CSF usually shows a normal glucose concentration with marginally elevated proteins but the white blood cell count is elevated predominantly with lymphocytes.

The diagnosis is based on isolation of L. monocytogenes from the blood cultures or occasionally from CSF cultures. The organism has rarely been identified on initial Gram staining. The issue of optimal antibiotics treatment is unclear and awaits clinical trials. The bacterium is sensitive to a wide variety of antibiotics but ampicillin and gentamicin are the recommended combination. ${ }^{4}$

Listeria rhombencephalitis is a treatable cause of brain stem encephalitis which presents in a characteristic bimodal pattern. Although the differential diagnosis of brain stem encephalitis is vast, this possibility should be reconsidered whenever progressive brain stem encephalitis is accompanied by sterile CSF culture. Our case exhibited signs of brain stem encephalitis which is a very rare manifestation of listeriosis along with signs of meningitis. This case also highlights the fact that brain stem encephalitis mainly affects otherwise healthy subjects.

S.A. Khan*

J.E. Pace

M.L. Cox
Department of Medicine for the Elderly, Watford General Hospital. Watford, Herts, UK.

*Present address: West Middlesex Hospital, Isleworth, Middlesex, UK.

\section{References}

1. Eck, H. Encephalomyelitis listeriaca apostematosa. Schweitz Med Wochenschr 1957, 87: 210-214.

2. Back, M.C. \& David, K.M. Listeria rhombencephalitis mimicking tuberculous meningitis. Rev Infect Dis 1987, 9: 130-133.

3. Callea, L., Donati, E., Faggi, L., Scalzini, A. \& Callea, F. Pontomedullary encephalitis and basal meningitis due to Listeria monocytogenes. Report of a case. Eur Neurol 1985, 24: 217-220.

4. Brightman, C.A. \& Dumbreck, A. Listeriosis. Br J Hosp Med 1989, 42: 366-370.

\section{Membranous nephropathy associated with diclofenac}

Sir,

Non-steroidal anti-inflammatory drugs (NSAID) have numerous effects on the kidneys ${ }^{\prime}$ including haemodynamic alterations, hyperkalaemia, acute interstitial nephritis, papillary necrosis, and the nephrotic syndrome associated with the 'minimal change' lesion. Memb ranous nephropathy has only rarely been reported in this context. ${ }^{2}$ We report a case of nephrotic syndrome due to. membranous nephropathy which occurred in a patient taking diclofenac and which resolved completely after withdrawal of the drug.

A 64 year old college lecturer presented with a 3 month history of leg swelling. He gave a history of subtotal thyroidectomy for hyperthyroidism 34 years previously and an episode of pancreatitis 4 years previously. He had taken diclofenac for non-specific arthritis for 18 months. On examination he had marked leg oedema. His blood pressure was $160 / 95 \mathrm{mmHg}$. Urinalysis showed heavy proteinuria but no haematuria. Twenty-four hour urinary protein excretion was elevated at $3.7 \mathrm{~g}$. Creatinine clearance was normal at $87 \mathrm{ml} / \mathrm{min}$ (serum creatinine $106 \mu \mathrm{mol} / \mathrm{l}$ ). Serum albumin was reduced at $25 \mathrm{~g} / 1$ whilst serum bilirubin, aspartate transaminase, and alkaline phosphatase were all normal. Chest $\mathrm{X}$-ray, abdominal ultrasound examination, and serum thyroxine were normal. Antinuclear factor, C3 and C4 components of complement, and serum protein electrophoresis were normal.

On renal biopsy 20 glomeruli were examined by light microscopy and showed mild mesangial increase but no capillary wall or other abnormality. Two glomeruli were examined by immunofluorescent microscopy and both showed diffuse granular capillary wall positivity for IgG. One glomerulus was examined by electron microscopy and showed numerous discrete subepithelial deposits in many of the capillary loops with no evidence of subendothelial or mesangial deposits. A diagnosis of early membranous glomerulonephritis (Stage 1 WHO classification) was made. Treatment with diclofenac was 